\title{
Project CROMATICA
}

Authors : L. KHOUDOUR(1), J.P. DEPARIS(1), J.L. BRUYELLE(2), F. CABESTAING(2), D. AUBERT(3), S. BOUCHAFA(3), S.A. VELASTIN(4), M.A. VICENCIO-SILVA(5), M. WHERETT(6).

Affiliations : (1) : INRETS-LEOST (French institute on Transport), (2) : University of Lille, (3) : INRETS-DART, (4) : King's College London, (5) : University College London, (6) : Molynx Limited.

Contact : L. KHOUDOUR, Phone : (33) 0320438336, Fax : (33) 0320438359, email : khoudour@terre.inrets.fr

\section{The Cromatica European Union Project}

The displacement of large numbers of people ("crowds"), specially at peak times, results in inevitable congestion and discomfort. Conversely, at periods with low traffic periods, mainly at evening and night, station premises are practically empty, creating an unsafe feeling in the mind of passengers, and favouring criminality or vandalism. To achieve a significant increase in the use of public transport in cities requires improvements in-: enhancement in the capacity to move people and quality of service. The CROMATICA project (CROwd MAnagement with Telematic Imaging and Communication Assistance) aims at developing tools for achieving these improvements.

The project's main user targets are-: managers and operations room personnel in stations/terminals and complete networks, network planners. From the brief discussion above, there is a clear need to automate the passengers monitoring process, both online and off-line. Such automation will have the greatest impact when integrated to existing control systems. The main telematic tool that can provide solutions for this problem is Image Processing :

- Measurement of crowd flows, mostly under "normal" conditions (to support demand-driven systems),

- Detection of abnormal crowd conditions (e.g. overcrowding, unexpected patterns of motion, queues),

- Detection of abnormal individual behavior or events that might affect services for a larger number of passengers (e.g. fall on the track, obstacles in the track, vandalism, personal attacks),

- Detection of unusual presence in forbidden areas (e.g. in stabled trains, blocking of exits/passageways).

\section{Project Participants}

The CROMATICA consortium is composed by 2 research centres (INRETS and CEA/LETI : France), one industrial (MOLYNX : Wales), 4 universities : King's College London and University College London (United Kingdom), Politecnico Di Milano (Italy), Université des Sciences et Technologies de Lille (France), 4 transport operators : RATP (France), London Underground (UK), British Airports Authority (UK), Azienda Trasporti Di Milano (Italy) and some other sponsoring partners. For 
more information on CROMATICA, please consult http://web.inrets.fr/UR/LEOST/PAGE.HTML.

In the framework of this conference, four different applications are chosen and described to illustrate the content of CROMATICA. These applications are :

- Automatic detection of falls on the tracks and intrusion in tunnels,

- Automatic detection of abnormal motion and stationarity in corridors,

- Automatic detection on some characteristics in the behavior of crowd,

- Routine data gathering.

\section{Fall on the Tracks and Intrusion Detection in Tunnels}

\subsection{Aim of the Application}

The aim of this application is to improve safety by detecting automatically people or objects falling on or crossing the tracks and people entering or exiting from the tunnels.

Through computer vision processing, the system to be developed determines and sends, in due time and via a satisfactory Human Machine Interface (HMI), alarms and the corresponding video image to the systems operators. Detection speed is a major improvement for safety and the minimisation of operation disruption. The system is built and installed on a site to inform in real time on the existence of incidents.

\subsection{Camera set-up}

In the current version of the system, to detect falls on the tracks, we propose to fix the cameras on the ceiling, aimed vertically so that the framing delineates clearly what is standing on the platform from what is above the tracks, so as to reduce the complexity of the software, and reduce the computing time accordingly.

To detect falls or intrusions, only the moving edges in the "tracks" part of the picture will trigger a warning. With this particular camera position, passengers bent over the edge are detected as possible incidents and potential accidents (which have actually happened), though other camera placements do not detect such incident scenarios and are being considered with the French operator RATP.

\subsection{Specific Algorithm}

According to the very hard working conditions (-uncontrolled lightening, changes of background, unknown moving objects) a specific robust algorithm has been designed to perform the detection of the moving edges. This algorithm is based on the analysis of the differences of gray level between successive frames in an original video sequence of images (Figure 1). Edge extraction, performed on the difference of successive images, allows to retain only the edges of moving objects in the frame. A refinement makes use of three successive images to avoid artefacts typical of other similar methods [1]. 


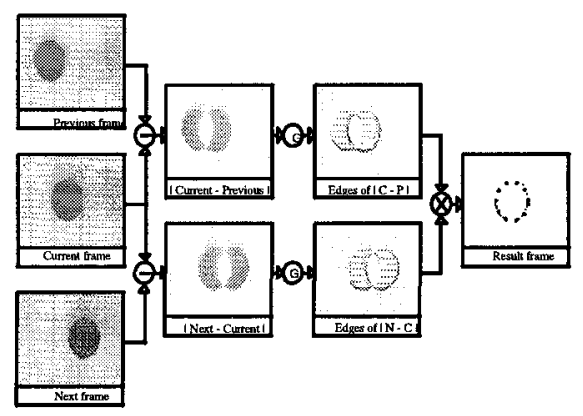

Fig. 1. Moving edges detector algorithm

Another particularly interesting feature of this algorithm is its ability to be easily implemented in hardware for real-time operation, thus reducing significantly the overall processing time. The moving edges are available as a binary image containing black and white pixels according to whether a moving edge has been detected or not.

\subsection{Example of Results in a test site}

The fall on the tracks and intrusion detection systems have been installed on an experimental station for test purposes. This test facility will be used to evaluate the detection reliability and the sources of false and nuisance alarms over a long period of time and under a variety of targets. For the moment, experimental results have been obtained using the laboratory prototype of the processor.

On figure 2, three images are shown for each example. The first image, on the left, is the original one, as seen by the camera. The second image shows the result of he motion detection process : the black parts of the image corresponds to the fixed areas of the scene, whereas white parts have been detected as areas affected by movement. In the third image, on the right, a segmentation step allows a precise detection of the moving bodies: the area corresponding to a moving body is surrounded by a polygonal shape.

The first two examples illustrate the performances of the fall detection system. The camera is set-up on the ceiling, with a small tilting. On figure 2.a, a man is falling on the tracks. We can see that he is clearly detected, and that he is seen as a single moving area by the system.

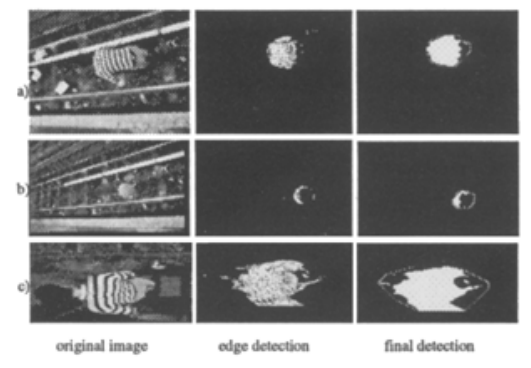

Fig. 2. Detection results

Multiple objects, such as sheets of paper, balls, handbags, etc... have been thrown at different speeds in the experimental surveillance system. Such small objects were 
always clearly detected. On figure $2 . \mathrm{b}$, we can see that a ball thrown on the tracks has been detected and segmented. A simple criterion on the size of the reconstructed silhouettes has proved to be very effective to discriminate between actual intrusions and nuisances caused by detected small objects.

Figure 2.c shows an intrusion in the forbidden area near the tunnel. A man is walking in the surveillance area. As for the fall detection, the set-up of the camera allows an efficient detection of the intrusion.

\section{Abnormal Motion and Stationarity in Corridors}

The aim of automatic detection of abnormal motion and stationary in corridors is to improve system operator workload, and safety by detecting automatically situations such as an aggression, a fight, a panic or an uneasiness, a dealer selling drug or an itinerant salesman, a threat (a madman, a terrorist, a fire, ...), an unattended object. In this paper, we describe the detection of counter-flow and stationarity in corridors.

\subsection{Counter flow Detection}

75\% of operators required such kind of detection for two different purposes. Concerning safety, a crowd "big enough" going in an opposite direction may indicate an incident. For quality of service, signalling a wrong way displacement may improve the management of the crowd.

For detecting moving people, techniques are generally based on the difference between a background image and the current one. The main difficulty is the way to update the reference image. To estimate motion, a matching approach [2] is widely used. However, there are some limitations (no global constraints, high sensibility to the "aperture" problem, sparse motion estimation, false match for low discriminated features). A spatio-temporal technique was also tried, but it works mainly thanks to a very specific camera position and only with sparse people.

After various experiments and evaluations, we chose to develop a differential technique. This technique is based on the hypothesis that the brightness of a particular moving point is constant in time. The Horn \& Schunk [3] algorithm gives a good flow field for this purpose, however the required computational time is not compatible with an on-line process. Thanks to some modifications (temporal filtering process) we overcome this problem and still get a good flow. Our preliminary experiments were made on various real situations (crowd density, camera positions and angles). We also made some trial at a zebra-crossing location. Even if the modified Horn \& Schunk algorithm is less accurate than the classical one, results are good enough to detect the various direction of motion (figures $3 \& 4$ ).

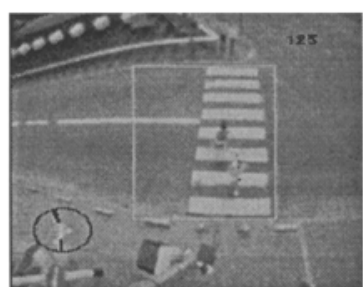

Fig. 3. Two people crossing a street in a reverse way.

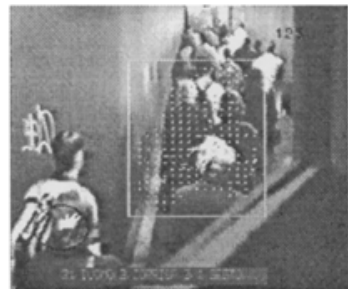

Fig. 4. A crowd turning in a corridor. The lines display the vector field. 
Our technique enables an "almost" global matching leading to a coherent flow field and decreases the "aperture problem" effect, giving us a reliable flow field. Moreover, the flow field is dense. However, there is still some false displacement vectors to filter before segmenting the image and computing the trajectories.

\subsection{Stationarity Detection in Corridors}

Roughly $77 \%$ of the public transport operators require such kind of detection for security reasons. Situations they want to detect through computer vision are people not able to get up after falling, dealers selling drugs, itinerant salespeople and objects left unattended. They are all characterised by people being stationary for a "long" period of time.

In the literature there is roughly nothing about stationarity detection. However, there is evidence it is done in some applications. They generally use a reference image to detect pedestrians. An analysis of their motion and sometimes shape gives the stationary ones. In this case a stationary person is detected if it does not display vertical head and body oscillations. However, most of these systems works under strong constraints about the camera position, the crowd density or do not take into account the fact that a stationary person is animated by movements.

In our case, thanks to a reference image dynamically updated, the system detects people and objects. From successive images it detects also all image areas corresponding to moving shapes. A difference between the moving areas and the foreground information gives stationary people and objects. An integration in the time scale of this detection enables to compute the duration of the stationarity. Above a given threshold, an alarm is triggered. To avoid any trouble generated by occlusion, the integration is performed only where there is no motion detected.

We currently evaluated the system on three video tapes (for a total of 50 minutes). Each one represents a different kind of situation (camera position and orientation, crowd density, movement of the stationary person). Results obtained are quite good (figures $5 \& 6$ ).

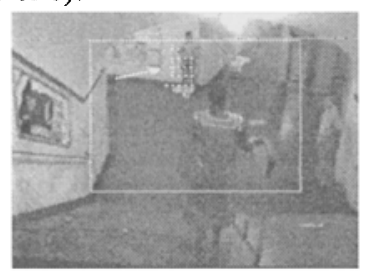

Fig. 5. One person is waiting in subway corridor. The crosses display stationary detection.

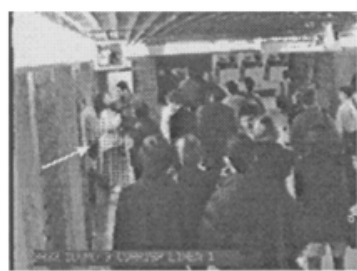

Fig. 6. Very crowded corridor. The back dots show the stationary person

We detected every stationary people (threshold at 2 minutes) and we did not get any false detection. However, such results must be confirmed on many more video tapes. Concerning the detection delay it varies drastically with occlusion. Currently, the higher the percentage of occlusion, the longer will be the detection delay.

\section{Potentially Dangerous Crowded Conditions}

In peak periods with high levels of crowding in a public transport system, it is vitally important that those managing a facility have timely information for the areas where problems are likely to arise even when small unexpected perturbations in the system 
occur. This leads to the concept of a Potentially Dangerous Situation that might not constitute an "incident", but that has to be monitored/controlled in order to prevent one. Within the scope of the CROMATICA project, the main objective is to develop and test image processing algorithms (working for a single video source) capable of detecting such potentially dangerous situations in various environments. A thorough study of users needs established the following main situations of interest :

- Excessive global occupancy (amount of used floor space),

- Excessive local occupancy: high crowding levels, non-homogeneous distribution,

- Rapid increase of either of the two above,

- Blocking of passageways by overcrowding and/or lack of motion, especially where areas of different capacity meet (e.g. hall-to-exit, corridor-toplatform),

- Abnormal queue lengths, shape or flow.

The main challenges are the ability to deal with high crowding levels with conventional camera positions (normally tilted, limited height), real-time potential and detection robustness.

There is little work reported in the open literature of solutions for analysing pedestrian scenes in crowded conditions (even for moderate crowding levels). Most of this type of work attempts to identify individuals from image shapes (heads, bodies). Thus their accuracy is severely limited when occlusion is present. Sometimes this problem is reduced by using vertically-mounted cameras, but this is not realistic for wide coverage in a public transport site. There has been little or no work elsewhere on identifying potentially dangerous situations involving crowds.

Techniques have been developed and tested with real-world data [4]. Detection of individuals is avoided. Instead, global image properties are measured and correlated to crowd parameters. These techniques can deal with moderate to high crowding levels in semi-confined environments (e.g. main halls, camera height $\sim 4 \mathrm{~m}$, camera inclination $\sim 45^{\circ}$ ). An example is given in figure 7.a where figure 7.b shows an exponential fit (thick line) between number of crowd pixels and actual number of people.

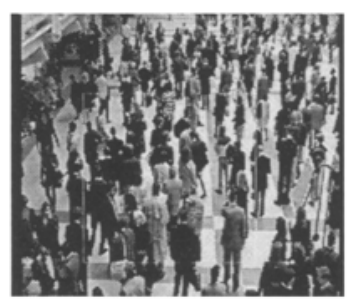

(a)

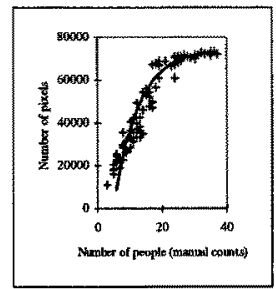

(b)

Fig. 7 a) Semi-confined environment (Liverpool St. Station, London. UK) ; b) Crowd pixels vs. number of people.

To deal with higher crowding levels and greater occlusion effects, new methods have been developed based on texture information illustrates how texture becomes richer with increasing crowding levels. Texture can be measured in any image region by observing grey level variations either in a statistical sense or in the frequency domain. 
A number of discrete ranges of occupancy is defined. A self-organising neural network is then trained to map a 16- or 24-valued texture vector into an occupancy range [5]. For global occupancy, a single texture vector (for the whole image) is computed. Typical results are shown in table 1. For example, $94 \%$ of the VH test set were classified correctly and $6 \%$ classified as $\mathrm{H}$. For local occupancy, texture vectors are computed for neighbourhoods around each pixel (figure 8).

\begin{tabular}{|c|c|c|c|c|c|c|c|c|c|}
\hline Resultsgetest Set & Classyatue & Range(people) & IL & & 1 & & & Moan & 0 \\
\hline Very Low (V) & & $\sqrt{0.15}$ & 94 & 6 & & & & 10.06 & 0.02 \\
\hline $\operatorname{Low}(L)$ & & $16-30$ & Ts & 34 & 30 & & & 1.20 & 0.06 \\
\hline Moderale (M) & & $31-45$ & & & 85 & & & 2.00 & 0.04 \\
\hline Hight( & & $446-50$ & & & & उat & & $\sqrt{3.06}$ & $\mid 0.02$ \\
\hline Very High (VM) & & $60+$ & & & & 6 & & $\sqrt{3.94}$ & 0.02 \\
\hline
\end{tabular}

Table 1 : Texture classification results (global occupancy) for 150 images
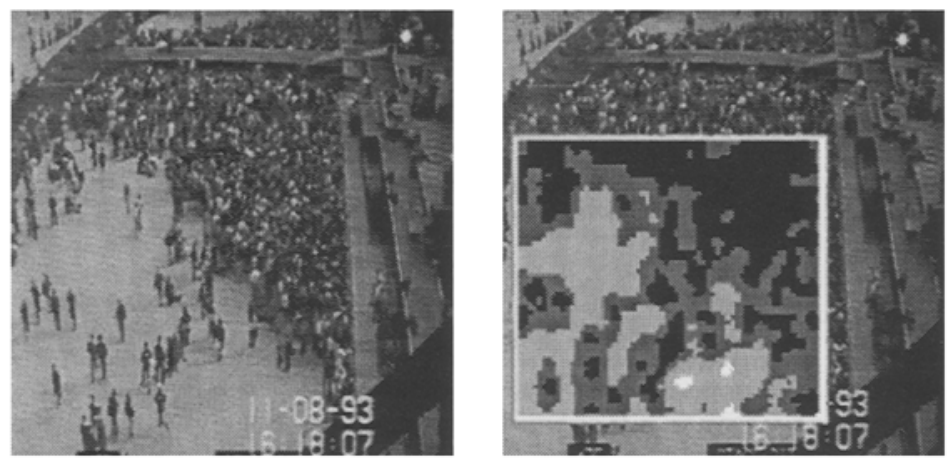

Fig. 8. Local occupancy through texture (whiter $\Rightarrow$ lower)

\section{Routine Data Gathering}

The routine data gathering system is an aid to system managers and planners and as such must provide appropriate information in a readily available and understandable format.

The user needs analysis survey undertaken at the beginning of the CROMATICA project identified user requirements for the following types of routine data gathering.

1. Relationship between signs and crowd movement: study how passengers watching signs affect crowd movement for optimal location of signs.

2. Relationship between station design and crowd movement: study how shapes of corridors, ticket areas entrances, etc. affect crowd movement for assisting in station layout design and / or modification.

3. For development of marketing strategies and medium term adjustment of schedules, surveys are required that count the following:

- The number of people waiting on the platforms,

- The number of people that get in the trains,

- The number of people coming out from the trains.

4. Detection of queues for tickets for improvement of Customer Service.

A system which provides the solutions for the user needs stated above will benefit the passengers and operators by improving safety (crowds can be prevented by 
intervention and better design), and - if the information is used to improve building design and layout - will provide a safer and more pleasant environment for the passengers.

At an early stage of the project, Molynx Limited's commercial people counting and monitoring system ("SensUs") was appraised regarding its capabilities and suitability for addressing the user requirements and new developments were identified. These were :

- Increase number of camera connections from a maximum of 4 to at least 32 .

- An improvement of the processing speed.

- A move towards standard system components.

- A compatible interface with CCTV systems.

From these requirements, an overall system architecture was designed.

A demonstrator has been defined with two main functionalities : "Occupancy monitoring and reporting" and "Queue monitoring and reporting". In most situations, analysis and reporting can be done off-line.

\section{Concluding Remarks}

In this paper we have briefly described four applications using image processing and aiming at an improvement of safety in public transport systems. These applications are part of the CROMATICA E.-U. project. Most of them are implemented and tested in life-situations.

\section{References}

[1] F. Cabestaing : "Détection de contours en mouvement dans une séquence d'images. Conception et réalisation d'un processeur cablé temps-réel" Thèse de l'Université des Sciences et Technologies de Lille. Janvier 1992.

[2] P. Vannoorenberghe, c. Motamed, J.M. Blosseville, J.G. Postaire, "Pedestrian flow estimation in urban environment by image processing", AATTE 95, Capri, June 1995.

[3] B.K.P. Horn, B.G. Schunck, "Determining optical flow", Artificial intelligence, 1981

[4] S.A. Velastin, J.H. Yin, A.C. Davies, M.A. Vicencio-Silva, R.E. Allsop, A. Penn (1994): "Automated Measurement of Crowd Density and Motion using Image Processing", 7th IEE International Conference on Road Traffic Monitoring and Control, 26-28 April 1994, London, UK, pp. 127-132.

[5] A.N. Marana, L da F. Costa, S.A.Velastin, R.A. Lotufo (1997): "Estimation of crowd density using image processing", IEE Coll. on Image Processing for Security Applications, London, March 10, 1997. 